\title{
Sobre a pluralidade de eventos e plurais essenciais ${ }^{1}$
}

\author{
On the plurality of events and essential plurals \\ Karina Veronica Molsing2 \\ Pontifícia Universidade Católica do Rio Grande do Sul - Porto Alegre - Rio Grande do Sul - Brasil
}

$\diamond$

\begin{abstract}
Resumo: Houve uma proliferação de estudos que atribuem um operador pluracional a leituras de eventos plurais de várias estruturas que antes recebiam uma abordagem não-pluracional, como, por exemplo, imperfectivos, progressivos e habituais (BERTINETTO, LENCI, 2012). $\mathrm{O}$ rótulo pluracional, entretanto, tem sido usado de forma inconsistente na literatura, gerando conflitos sobre a natureza da pluracionalidade e como figura no quadro maior de pluralidade de eventos. $\mathrm{O}$ foco deste trabalho é o pretérito perfeito composto no português brasileiro, que também já recebeu o tratamento pluracional. Neste artigo, sugere-se uma abordagem que dê conta da pluralidade sem recorrer à pluracionalidade. A semântica de argumentos plurais mostra que nenhuma condição proposta para a definição de pluracionalidade se sustenta na definição do pretérito perfeito composto. Dado esse insight, assuma-se plurais essenciais, de Schein (1993), para poder reformular a questão da pluralidade sem referência à pluracionalidade, mantendo, ao mesmo tempo, as intuições de uma semântica baseada em eventos.
\end{abstract}

Palavras-chave: Pretérito perfeito composto; Eventos; Plurais; Semântica neo-davidsoniana; Pluracionalidade

\begin{abstract}
There has been a proliferation of studies attributing pluractional operators to plural event readings of a variety of structures that have previously been treated in a non-pluractional manner, such as imperfectives, progressives and habituals (BERTINETTO, LENCI, 2012). The pluractional label, however, has been used in a non-systematic manner across the literature, generating conflicting conceptions of what exactly pluractionality is and how it figures into the larger picture of event plurality. The focus of this paper is the present perfect in Brazilian Portuguese, which has also recently been receiving the pluractional treatment. The present paper suggests an exercise in accounting for plurality without appealing to pluractionality. The semantics of plural arguments show that none of the conditions proposed in the definition of pluractionality can be maintained for the present perfect. Given the insight provided by plurals, we assume essential plurals, by Schein (1993), in order to recast the issue of plurality without reference to pluractionality, while maintaining intuitions captured by event-based semantics.
\end{abstract}

Keywords: Present perfect; Events; Plurals; New-davidsonian semantics; Pluractionality

\section{Introdução}

$\mathrm{O}$ pretérito perfeito composto (doravante, $P P C$ ) no português brasileiro (doravante, $\mathrm{PB}$ ) se refere à estrutura composta do verbo auxiliar ter e do particípio passado. Quando comparado com as estruturas correspondentes em espanhol e inglês, observamos que o PPC possui um sentido distinto, como se vê em (1).

\footnotetext{
$\overline{1}$ O presente trabalho foi realizado com o apoio da CAPES/FAPERGS. Uma versão ampliada deste capítulo foi publicada em inglês em Molsing e Ibaños (2013).

2 Bolsista de pós-doutorado da Pontifícia Universidade Católica - Rio Grande do Sul (PUCRS).
}

(1) a. Português: Maria tem ido à praia.

b. Espanhol: Maria ha ido a la playa.

c. Inglês: Maria has gone to the beach.

O PPC necessariamente significa que houve várias ocasiões em que a Maria foi para a praia. Em espanhol e em inglês, o significado do perfeito é, no mínimo, de caráter existencial, de modo que ocorreu pelo menos um evento de Maria ir para a praia antes de agora. Qualquer outra inferência sobre quanto tempo faz ou se a Maria ainda está lá pode variar com o contexto. O sentido existencial para o PPC não é possível. É interessante 
notar que esta leitura já foi disponível na história recente (BOLÉO, 1936; SUTER, 1984).

Em outras palavras, o PPC expressa uma repetição obrigatória, indefinida e não-contável e, desta forma, somente é compatível com advérbios de frequência vaga, conforme o exemplo em (2). Esta incompatibilidade do PPC com modificações cardinais reflete um comportamento semelhante ao imperfeito. O PPC também é incompatível com advérbios que marcam o tempo passado, conhecido como o "enigma do presente perfeito" (present perfect puzzle, KLEIN, 1992, 1994).

(2) a. Maria tem ido à praia todos os dias/*ontem/*uma vez/*três vezes

b. Maria ia à praia todos os dias/*ontem/*uma vez/*três vezes

Com esses dados, fica claro que o problema não pode ser contornado através da incorporação de algum elemento com o significado de pelo menos duas ou mais ocorrências (AMARAL, HOWE, 2005), visto que o PPC não pode ser modificado por qualquer número definido. A estrutura aceita apenas modificações de frequência indefinida.

Embora a especificidade principal do PPC seja o significado de iteratividade expresso com verbos de ação, o PPC também pode expressar situações durativas, como em (3).

(3) John tem estado doente desde fevereiro.

Entretanto, a maioria das teorias contemporâneas para o PPC não abordam a leitura durativa. Portanto, com foco na iteratividade, existem pelo menos três possibilidades teóricas na literatura para descrever e explicar a origem desta repetição: i) o auxiliar (ALMEIDA, 2006; JACINTO, 2007); ii) uma combinação do auxiliar e do particípio passado (GIORGI, PIANESI, 1997; SCHMITT, 2000)33; e iii) a estrutura como uma unidade semântica. Vamos concentrar neste último tipo de abordagem, particularmente as teorias que propõem que esta unidade semântica seja de natureza pluracional, conceito este a ser elucidado na próxima seção. O objetivo, então, é apresentar uma revisão crítica de dois tipos de abordagens para o PPC, com foco na natureza pluracional da estrutura.

A próxima seção apresenta um estudo sobre o PPC de um dialeto brasileiro que parece fugir do PB padrão. A seção 2 relata um estudo diacrônico com base num corpus de português. Assumindo-se o desejo de construir uma teoria composicional para o PPC, e, portanto, sem recorrer a um operador pluracional, a seção 3 aponta alguns conceitos fundamentais sobre objetos e eventos no contexto de plurais para desenvolver outro caminho para o PPC. Tais conceitos seriam necessários para uma abordagem composicional do PPC dentro de uma teoria de eventos. A última seção conclui.

\section{Um PPC restrito}

Cabredo-Hofherr et al. (2010) examinam o PPC em um dialeto nordestino brasileiro que é altamente restrito, quando comparado com o PPC padrão, seja na variedade do português brasileiro ou do português europeu. Além das restrições usuais em relação à referência a eventualidades ${ }^{4}$ do tempo passado, as condições nas quais as leituras durativas podem surgir neste dialeto nordestino (doravante, NE-PPC) são muito limitadas. E os contextos nos quais a leitura plural é aceitável também são limitados. Por exemplo, as sentenças abaixo, tiradas de Cabredo-Hofherr et al. (2010), embora aceitáveis no PB padrão, sugerem que o NE-PPC requer mais do que uma simples iteração para obter uma leitura aceitável.

(4) a. \#Maria tem tossido sem parar / muito desde ontem.

b. \#Desde que ele conheceu a Maria, ele tem esperado as cartas dela.

c. ??O nível do rio tem aumentado muito.

d. O número de alunos tem aumentado.

e. Eu tenho feito as camas desde segunda-feira.

Além de expressar uma pluralidade de eventos, o uso adequado do NE-PPC também requer que tais eventos sejam descontínuos. É por isso que o uso de predicados stagelevel é restrito à interpretação não-homogênea, como em (5a), diferente do PB padrão que aceita leituras realmente durativas, como em (5b) (ILARI, 2001).

(5) a. A biblioteca tem ficado fechada (nos domingos).

b. A biblioteca tem ficado fechada (desde o início da greve).

A possibilidade de obter leituras frequentativas, habituais e incrementais sugere que esta construção possui um caráter pluracional ${ }^{5}$. O NE-PPC apresenta

\footnotetext{
3 Ver Molsing (2010) para uma discussão dos tipos (i) e (ii).

4 O termo "eventualidade" vem de Bach (1986) que abrange todos os tipos de classe verbal. Para uma apresentação detalhada dos pressupostos básicos comuns aos estudos discutidos aqui - tempo, aspecto, eventos e pluralidade - ver Molsing (2010).

5 Lasersohn (1995) propôs a pluracionalidade como um tipo de pluralidade verbal que é frequentemente marcada por afixos morfológicos no verbo. A pluralidade pode se referir a múltiplas ações, participantes, tempos ou lugares. Entretanto, esse tipo de pluracionalidade também pode ser expressa lexicalmente nas línguas como inglês com expressões do tipo over and over (repetidamente) e keepx-ing (continuarx-ndo). Lasersohn define marcadores pluracionais com o sentido converso de junto (together). Ao invés de significar sobreposição temporal, a pluracionalidade significa separação, ou não-sobreposição. A porção do significado pluracionalque se refere a nãosobreposição pode ser negada para leituras contínuas. Lasersohn entende que os marcadores pluracionaisse aplicam a grupos de eventos, da mesma forma que os sintagmas nominais plurais denotam grupos de indivíduos.
} 
certas propriedades características de outras perífrases verbais, tais como andar+gerúndio, e, desta forma, Cabredo-Hofherr et al. (2010) assumem que tais perífrases incorporam operadores pluracionais no seu significado. Por exemplo, as autoras afirmam que não há efeitos de multiplicação em argumentos singulares indefinidos. Ver os exemplos em (6).

(6) a. \#Tenho comido uma maçã.

b.\#Ando comendo uma maçã.

Esse tipo de exemplo também é restrito no PB padrão. Entretanto, se os eventos (ou subeventos) são considerados repetíveis, é possível obter multiplicação. Ver os exemplos em (7).

(7) a. Tenho tomado um vinho maravilhoso.

b. Maria tem encontrado um erro no manuscrito.

Pelo menos no PB padrão, assumindo um tipo de erro, a restrição em (7b) não se deve necessariamente ao significado do PPC, mas ao significado de verbos que denotam eventos de consumo, criação ou destruição, etc. Além disso, com alguns argumentos plurais, surgem efeitos de distribuição, como em (8) abaixo.

(8) a. Tenho comido maçãs.

b. Muitas / \#Vinte pessoas têm morrido na guerra.

O PB padrão é um pouco menos restrito com respeito a advérbios que expressam uma cardinalidade vaga, como se vê em (9a-b). As leituras restritas em (9a) seriam aceitáveis no PB padrão, enquanto os advérbios cardinais definidos, como em (9b), são inaceitáveis em ambas as variedades. Por outro lado, ambas as variedades, PB padrão e NE, aceitam advérbios de frequência cíclica e distributivos, como em (9c-d).

(9) a. Eu tenho visto \#várias / \#muitas vezes a sua irmã.

b. \#Eu tenho visto três vezes a sua irmã.

c. Tenho visto a sua irmã todo dia.

d. Ela tem ligado uma vez por semana.

As autoras utilizam esses exemplos com o objetivo de mostrar que a mera pluralidade de eventos não é suficiente para o uso correto do NE-PPC. A repetição deve ser distribuída uniformemente dentro do intervalo de referência. Consequentemente, os dados para o NE-PPC levam Cabredo-Hofherr et al. (2010) a propor que esta estrutura deva ser representada por um operador semelhante às perífrases verbais como andar+gerúndio, dadas as semelhanças nas leituras frequentativas e habituais, bem como a estrutura interna descontínua do intervalo de referência.
Ao mesmo tempo em que o emprego do operador pluracional é confessadamente um caminho nãocomposicional para a representação do significado da estrutura do NE-PPC, as autoras afirmam que ainda não foi proposta na literatura uma análise composicional. Cabredo-Hofherr et al. (2010) acreditam que a busca pela composicionalidade deve ser focada numa investigação aprofundada do auxiliar ter porque a língua portuguesa é a única entre as românicas que utiliza ter ao invés de haver ${ }^{6}$. Questiona-se, entretanto, como uma análise diacrônica ou sincrônica de ter versus haver explicaria as diferenças nas restrições entre o PPC do dialeto nordestino e do PB padrão.

\section{Um estudo diacrônico do PPC pluracional}

Vários estudos diacrônicos mostraram que pelo menos desde o século XV, ter e haver eram intercambiáveis, embora o primeiro ocorria com mais frequência do que o segundo nas perífrases verbais (ALMEIDA, 2006; AMARAL, HOWE, 2012). Com o passar do tempo, o uso de ter nas construções perifrásticas aumentou muito em comparação com haver, e os usos de haver se tornaram mais restritos em geral, não somente como auxiliar (LUNGUINHO, 2011). A coexistência da construção resultativa com ter durante este tempo levou a ambiguidades, particularmente quando não está claro se há concordância com o objeto direto ou não. Veja os exemplos em (10).
(10) a. Tenho as camas feitas.
b. Tenho o livro xerocado.

Desta forma, a explicação diacrônica é que certas ambiguidades provocaram uma mudança semântica via um processo de gramaticalização (HOPPER, 1991; HOPPER, TRAUGOTT, 1993). O que varia entre uma abordagem e outra é a definição dessas ambiguidades e como condicionam uma nova interpretação semântica.

Argumentou-se que a ambiguidade estrutural exemplificada acima entre a construção resultativa e o PPC levou a uma perda de concordância entre o adjetivo de verbos transitivos e o objeto direto (Said Ali 1964). Portanto, as abordagens diacrônicas para o PPC focam no processo de gramaticalização de ter e haver como verbos principais para auxiliares. A ideia geral é que o uso de ter aumenta na medida que o uso de haver diminui e o significado lexical de ter ocasiona novas leituras para o PPC. Ao mesmo tempo, as ambiguidades estruturais com

\footnotetext{
${ }^{6}$ Espanhol haber, francês avoir e italiano avere. Francês e italiano também possuem perfeitos com ser, utilizando être e essere, respectivamente.
} 
as construções resultativas provocam uma reinterpretação do PPC. Consequentemente, o uso de construções resultativas fica reduzido.

Amaral e Howe (2012) trabalham a partir de conceitos de estudos anteriores relativos à natureza pluracional do PPC. Também fazem uso de dados diacrônicos com foco no significado antigo do PPC, como uma construção adjetival resultativa, e sua coexistência com a interpretação plural. Os autores assumem que o PPC é uma unidade semântica cujo significado é representado por um operador pluracional. Ao invés de focar somente na contribuição lexical de ter, propõem caracterizar a emergência deste significado através de uma série de elementos e ambiguidades sintáticas e semânticas coexistentes.

A ambiguidade sintática se refere ao contexto em que há um objeto direto e um particípio singular e masculino tal que a estrutura pode ser entendida como um resultativo ou o PPC. Amaral e Howe argumentam que a leitura plural emerge quando o objeto direto é um pronome indefinido, um quantificador universal ou um substantivo massivo. Veja abaixo o seu exemplo do século XVI (2012, p. 40).

\section{(11) em tudo o que escrito tenho, o tenho mizclado.}

A ambiguidade se refere ao quantificador tudo que toma o escopo sobre os trabalhos escritos ou sobre os eventos de escrever. Portanto, Amaral e Howe afirmam que a emergência da leitura iterativa ocorre dentro do sintagma verbal e o resultado da pluralidade do objeto direto desencadeia uma distribuição sobre uma pluralidade de eventos.

Em suma, a ocorrência crescente de ambiguidades sintáticas e semânticas como a descrita acima gradativamente levou a uma reanálise do PPC tal que a pluralidade de eventos entrou no significado semântico do PPC. Aparentemente começando por verbos de atividade com objetos plurais ou massivas, ou quantificadores universais, como em (11), esta reanálise levou a uma ampliação deste novo significado para outras classes verbais, inclusive as com objetos singulares e substantivos contáveis, tais como accomplishments, bem como as sem objetos, como estados.

Com respeito aos plurais na posição de sujeito, Amaral e Howe afirmam que os $\mathrm{SNs}$ cardinais são proibidos, mas os plurais nus e outros SNs quantificados podem contribuir para uma leitura de eventos plurais. Um de seus exemplos está repetido em (12a), no qual a restrição parece ser devido à natureza única de verbos como morrer e nascer e não ao PPC.

(12) a. ??Dez animais têm nascido nesta rua ${ }^{7}$.

b. Dez animais têm nascido nesta rua todo dia.

c. ??A Maria tem nascido nesta rua. (12b) mostra que a cardinalidade do sujeito pode ser aceitável num contexto de frequência. Além disso, se alterarmos o cardinal para Maria, como em (12c), o resultado também não é aceitável visto que, sob circunstâncias normais, a Maria não nascer mais de uma vez. Portanto, acredita-se que as restrições se devem à natureza da eventualidade subjacente e à maneira como os sujeitos podem participar dos eventos, e não à cardinalidade do sujeito ou à estrutura do PPC.

Os autores afirmam ainda que as leituras de eventos plurais desencadeadas por sujeitos plurais não são necessariamente distributivas, e podem potencialmente resultar em uma interpretação de eventos simultâneos, que não é compatível com o significado do PPC. Entretanto, os exemplos utilizados pelos autores para ilustrar este argumento são do passado simples em inglês. Veja o exemplo abaixo (2012, p. 48).

(13) [PL Peter, Paul and Mary] washed a car. Peter, Paul e Mary lavaram um carro.

Evidentemente, em (13), as leituras possíveis são que Peter, Paul e Mary ou lavaram de forma coletiva um único carro, ou lavaram individualmente diferentes carros ao mesmo tempo. Consideremos o argumento utilizando o PPC, conforme em (14).

\section{(14) Peter, Paul e Mary tem lavado um carro.}

Em (14), a leitura coletiva significa que há eventos de lavar, que são uniformemente distribuídos dentro do intervalo de referência, de modo que Peter, Paul e Mary participam de forma coletiva em cada evento de lavar. Diferentemente de (13), a leitura alternativa em (14) não poderia ser de múltiplos eventos simultâneos de lavar carros diferentes em uma única ocasião. Independentemente de ser um único carro ou diferentes carros sendo lavado, o sujeito plural não interfere com a distribuição de eventos de lavar, que surge da própria estrutura do PPC. Entretanto, o uso de um sujeito plural altera a forma como os indivíduos participam dos eventos. Enquanto os indivíduos de objetos (plurais) são distribuídos sobre (sub)eventos, os sujeitos plurais criam uma relação vaga com os objetos plurais referente a sua participação dos eventos. (14) pode, portanto, ser entendido conforme a seguir: há uma pluralidade de eventos de lavar distribuídos no intervalo de referência e Peter, Paul e Mary eram os lavadores e o carro (ou o tipo de carro) foi lavado. A natureza desta participação é vaga e não explicitamente distributiva. Isto é, qualquer combinação de membros do sujeito plural pode ter participado dos eventos de lavar. Isso significa que Peter e Paul pode ter participado de uma lavagem, Paul e Mary

\footnotetext{
7 Exemplo de Amaral e Howe (2012, p. 27).
} 
de outra, os três de outra, apenas Peter de outra, e assim por diante. É importante notar que essa visão deixa em aberto a possibilidade de pelo menos algumas lavagens simultâneas, o que entra em confronto com o significado de pluracionalidade. Entretanto, é um contexto permitido pelo PPC no PB padrão e que passou despercebido devido a uma falta de consideração dos plurais na posição de sujeito.

Assim, enquanto o papel dos sujeitos plurais na emergência de uma leitura iterativa do PPC não está claro, entende-se que contribuem uma referência plural a eventos. Em outras palavras, a ideia apresentada aqui é que o PPC faz uma referência plural a eventos assim como fazem os argumentos plurais. Entretanto, cada tipo de plural difere em como se dá tal referência, isto é, em como os eventos são individuados. Com a inclusão de cada plural, seja de natureza nominal ou verbal, altera-se a divisão e a interpretação do intervalo do evento. Este argumento será aprofundado no contexto da abordagem de Schein (1993) para plurais, na seção 4.

Em suma, parece adequada a explicação de Amaral e Howe (2012), no seu estudo diacrônico, para a emergência da leitura iterativa do PPC e de como as ambiguidades sintáticas e semânticas contribuem para tal interpretação. Entretanto, o mesmo auxiliar e o potencial para ambiguidades existe em outros contextos perifrásticos, como, por exemplo, no mais-que-perfeito. Curiosamente, essas mesmas condições de ambiguidade com objetos plurais não levaram a leituras plurais nas outras construções com ter como auxiliar. Ver (15) abaixo.

(15) a. Tinha as camas feitas.

b. Tinha o livro xerocado.

Os fatores que motivam a mudança também estariam presentes para o mais-que-perfeito ${ }^{8} \mathrm{e} o$ futuro do presente composto, bem como os tempos subjuntivos. Porém, tais mudanças não ocorreram, visto que o significado resultativo/anterior permanece para a maioria das estruturas com o auxiliar ter.

Assim, dado o fato de a leitura plural ser necessária apenas quando o auxiliar está no tempo presente, a relação entre ter e a fonte da pluralidade deve ser indireta. Tal diferença, entre outras, motivaria uma reanálise de ter $+\mathrm{PP}$ à luz da semântica do tempo presente.

Desta forma, a afirmação de que o PPC contemporâneo pode ser descrito como um operador pluracional na mesma linha de outras perífrases verbais como andar+gerúndio seria adequada pressupondo-se que o PPC seja uma unidade sintático-semântica. Crucialmente, também seria adequada pressupondo-se que uma interpretação de eventos sobrepostos/simultâneos seja impossível, mesmo na presença de sujeitos plurais, o que parece ser incorreto. Portanto, se aceitarmos esta interpretação e se considerarmos a contribuição do tempo presente no significado contemporâneo do PPC, deve-se contemplar outro caminho.

Com base na discussão apresentada até aqui, buscamos um entendimento da iteratividade no PPC sem recorrer ao conceito de pluracionalidade, que, além de não-composicional, não captura todas as intuições possíveis com essa estrutura. Assim, pretende-se analisar a pluralidade do PPC com foco na contribuição dos argumentos plurais. Uma teoria do PPC não seria completa sem também uma investigação da semântica do auxiliar ter no tempo presente, bem como do significado de anterioridade do particípio passado. Neste trabalho, entretanto, abordaremos apenas as relações de pluralidade em contextos com argumentos plurais ${ }^{9}$.

\section{Sobre plurais essenciais}

O sentido principal do operador pluracional, utilizado pelos estudos levantados nas seções anteriores para definir o PPC, é o de a não-sobreposição de eventos, que são distribuídos uniformemente no intervalo de referência. Entretanto, ao discutir a natureza dos plurais numa teoria semântica com base em eventos (LINK, 1983; LANDMAN, 2000; LASERSON, 1995), podemos chegar a um entendimento melhor de exemplos como (16) e (17) abaixo.

(16) João tem alugado um carro nos últimos meses.

(17) Os executivos têm alugado um carro nos últimos meses.

Em (16), o significado é de múltiplos eventos de alugar por João, enquanto em (17), o plural executivos parece expressar uma relação vaga sobre como os executivos participam dos eventos de alugar. É possível que haja pelo menos alguns aluguéis simultâneos entre todos os eventos de alugar, dado o sentido de alugar assim como a possível leitura de tipo de um carro. Esta interpretação não entra em conflito com o significado do PPC, embora entre em conflito com o significado de pluracionalidade. Consequentemente, pode-se atribuir a interpretação pluracional do PPC à singularidade/ pluralidade do sujeito e não à própria estrutura do PPC, logo sugerindo uma gama mais ampla de sentidos do PPC.

Propõe-se, então, recorrer a uma análise de objetos e eventos plurais na linha de Schein (1993), que opta por um tratamento de plurais que depende principalmente de uma mereologia de eventos (i.e. uma relação

\footnotetext{
8 Ainda mais marcante é o fato de o auxiliar ter a forma imperfectiva e, ainda assim, é incompatível com a iteratividade obrigatória do PPC.

9 Para uma proposta que aborde a contribuição de tempo e aspecto no significado do PPC, ver Molsing (2013).
} 
parte-todo entre um evento e seus subeventos) e de relações temáticas entre os participantes e seus respectivos eventos. Nas sentenças com argumentos plurais, a pluralidade é sempre deslocada para o evento. As leituras distributivas envolvem uma pluralidade de eventos, cada um com o seu próprio agente, enquanto as leituras coletivas envolvem um evento em que os subeventos se restringem a um único agente.

Schein afirma que sempre há vaguidade quando se tem mais de um plural na sentença porque, "é isso que os torna plurais essenciais" (1993, p. 16). Veja (18), por exemplo.

(18) Três meninos convidaram quatro meninas para a festa.

A vaguidade aqui se deve à relação cumulativa entre os dois plurais na sentença. Isto é, existe uma referência de segunda ordem para um evento composto de subeventos de convidar e os agentes desses subeventos são os três meninos e os pacientes são as quatro meninas. Para cada um desses subeventos, alguns dos meninos convidam ou de forma distributiva ou de forma coletiva algumas das meninas.

Passamos agora para os "plurais essenciais", o foco do trabalho de Schein (1993), conforme mostra o exemplo (19).

\section{(19) Choveram pedras.}

Neste caso, não se pode dizer que um grupo de pedras é tal que ele choveu ou que cada pedra é tal que ela choveu. A sentença significa apenas que as pedras são tal que elas choveram. Ao invés da sentença ser sobre algum objeto plural, neste caso as pedras, é sobre um evento de chuva, e cada uma daquelas pedras participa daquele evento.

Pode-se ver que, para Schein, a representação de plurais requer não somente uma referência a eventos, mas é uma referência neo-davidsoniana ${ }^{10}$ a eventos e a papéis temáticos. Em geral, os papéis temáticos expressam a natureza da relação entre o objeto plural e o evento. $\mathrm{Na}$ visão de Schein, o papel temático faz parte do significado do próprio plural. Desta forma, a predicação plural se reduz a predicação singular de objetos e eventos onde a própria pluralidade é atribuída para a relação que existe entre os objetos e o evento. Tal redução, chamada de "decomposição", é necessariamente aplicada a cada posição argumental com um plural (SCHEIN, 1993, p. 4).

Nesse exemplo, vê-se a necessidade de incorporar uma mereologia de eventos, pois a participação de cada objeto singular é considerada um subevento, e então, todas essas participações individuais são somadas e ligadas a

\footnotetext{
${ }^{10}$ Para uma apresentação da visão neo-davidsoniana de eventos, ver Molsing (2010).
}

um evento maior. Crucialmente, a mereologia de eventos também permite a referência cruzada de eventos. Por exemplo, se acrescentarmos um advérbio no exemplo (19), e.g., "Caoticamente, choveram pedras", não significa que a queda de cada pedra é caótica, mas a soma de todas as pedras caindo é caótica. Esta relação parte-todo envolve um quantificador distributivo sobre partes de eventos. Em outras palavras, a distribuição sobre indivíduos é entendida como a distribuição sobre eventos.

Em suma, a intuição de Schein em relação à distribuição sobre indivíduos e sobre eventos é a noção fundamental que motiva o tratamento que Amaral e Howe (2012) dão para a emergência do significado de pluralidade na estrutura do PPC, conforme discutido na seção 3. Ampliamos essa ideia para incluir a concepção de todos os plurais como predicados, seguindo Schein (1993). Trata-se, especialmente, dos plurais essenciais, que fazem uma referência plural e indefinida a eventos. A ideia central é que algumas coisas (juntas) podem denotar um predicado plural sem que essas coisas sejam consideradas um conjunto. Tais eventos plurais são representados por um termo plural de segunda ordem que não é contável e significa "alguns eventos" ou "uma pluralidade de eventos que pode ou não ser tão densa como uma massa eventiva" (SCHEIN, 1999, p. 83). Percebe-se que esta definição expressa uma pluralidade "fraca", sendo, portanto, compatível com valores plurais e singulares. Assuma-se esta visão de plurais para explicar as leituras do PPC, não somente com sujeitos plurais, mas também com argumentos plurais cardinalizados, ambos os quais não foram abordados no trabalho de Amaral e Howe (2012).

Segue um resumo das principais características desta visão:

1. Predicados básicos de pluralidade "fraca": substantivos e verbos não-marcados são compatíveis com valores tanto plurais como singulares.

2. Uma mereologia de eventos: o domínio de eventos possui uma estrutura parte-todo.

3. Decomposição neo-davidonsiana: a predicação plural se reduz a predicação singular para objetos e eventos.

4. Separação do papel temático: os objetos e eventos se relacionam através de conjuntos com papéis temáticos. A natureza da relação temática é importante para dar conta dos diferentes tipos de leituras. Para Schein (1993), isso significa que a pluralidade de sintagmas nominais em leituras coletivas, distributivas e cumulativas se desloca para o evento, resultando em uma pluralidade de eventos ou subeventos.

5. Referência cruzada de eventos: existe uma anáfora descritiva para ligar os subeventos ao evento maior. 
Nesta abordagem, o próprio PPC seria um tipo de plural essencial, porém de natureza verbal. Com argumentos singulares, o PPC contribui uma pluralidade de eventos com respeito a ocasiões diferentes. Com objetos plurais, como em (20), trata-se da leitura distributiva, com ocorrências em ocasiões diferentes com um livro diferente em cada ocasião.

\section{(20) O aluno tem lido os livros}

A presença de um sujeito plural acrescenta mais um nível de pluralidade dentro das ocasiões. É interessante perceber que a leitura que surge nesses casos é o mesmo tipo de quando há dois nominais plurais na mesma sentença, i.e. cumulativa, como em (21a) abaixo. Não há informações sobre quais professores escreveram quais artigos.

(21) a. Cinco professores escreveram cinco artigos.

b. Cinco professores têm defendido uma teoria.

Em (21b), os sujeitos plurais junto com o PPC desencadeiam uma relação de cumulatividade entre os cinco professores e os eventos de defender uma teoria. Nesse caso, há uma referência cruzada de eventos. Assumindo Schein, o exemplo seria parafraseado como "existem alguns eventos de defender, e $a_{i l}{ }^{11}$, os professores são os defensores e a teoria é defendida". Isso significa que os professores não são necessariamente distribuídos uniformemente nos eventos de defender, mas podem ser combinados de várias formas, e cada professor possui seu próprio subevento de defender a teoria. Imagine um congresso com sessões paralelas e dois professores estão fazendo apresentações em que defendem a mesma teoria no mesmo horário em salas diferentes. Portanto, é bem possível ter eventos sobrepostos de defesas da teoria sem entrar em conflito com o significado do PPC. Isto é, não há compromisso semântico no significado dessa estrutura sobre como os subeventos se organizam dentro do intervalo de referência. Como o sentido de sobreposição bem como de não-sobreposição sempre são possibilidades, não devem fazer parte do significado básico do PPC. O mesmo raciocínio vale para todas as classes verbais.

\section{Conclusão}

Motivado pela possibilidade de uma abordagem composicional, o presente trabalho apresentou uma revisão crítica de alguns trabalhos contemporâneos sobre o PPC que utilizam o conceito de pluracionalidade para descrever e explicar a leitura de iteratividade. A partir

\footnotetext{
${ }^{11}$ Ali (ou there, em SCHEIN, 1993) representa a anáfora descritiva que liga alguns eventos com os argumentos plurais em questão.
}

desta revisão, procurou-se investigar de forma mais aprofundada o papel dos plurais no significado desta estrutura. Como sugerido por Amaral e Howe (2012), seguindo Schein (1993, 1999), os objetos plurais desencadeiam uma distributividade sobre a pluralidade de subeventos dentro de ocasiões.

Propôs-se aqui que os sujeitos plurais também contribuem para um melhor entendimento do PPC, embora a pluralidade seja de outra natureza. Diferente da sugestão por Amaral e Howe (2012), não há uma restrição específica ao PPC sobre argumentos plurais cardinalizados. Pelo contrário, quaisquer restrições se devem à natureza da eventualidade subjacente em relação aos argumentos plurais e não ao próprio PPC. De outro modo, a presença de sujeitos plurais resulta em uma relação vaga com os eventos plurais do PPC. Portanto, assumiu-se aqui uma visão mais ampla dos plurais de Schein para poder dar conta da gama de significados expressos pelo PPC. Nesta perspectiva, a estrutura do PPC se comporta como um "plural essencial" que contribui um significado de eventos plurais, indefinidos e não-contáveis. Ao acrescentar objetos plurais, obtém-se uma leitura distributiva e, ao acrescentar sujeitos plurais, obtém-se uma vaguidade sobre as participações dos eventos.

\section{Referências}

ALI, Manuel Said. Gramática histórica da língua portuguesa. 3. ed. São Paulo: Melhoramentos, 1964.

AMARAL, Patricia; HOWE, Chad. The semantics of the Portuguese present perfect and the grammaticalization of ter/ haver. In: The $8^{\text {th }}$ annual Ohio state university symposium on Hispanic and Luso-Brazilian literatures, linguistics and culture. Ohio, 2005.

AMARAL, Patricia; HOWE, Chad. Nominal and verbal plurality in the diachrony of the Portuguese present perfect. In: LACA, B.; CABREDO-HOFHERR, P. (eds.). Verbal plurality and distributivity. Berlin: de Gruyter, 2012. p. 25-52.

ALMEIDA, Erica Sousa de. A formação de perífrases verbais no português: um processo diacrônico. Master's thesis, Universidade Federal do Rio de Janeiro, 2006.

BOLÉO, Manuel de Paiva. O pretérito e o perfeito em português, em confronto com as outras línguas românicas. Coimbra: Imprensa da Universidade, 1936.

CABREDO-HOFHERR, Patrícia; LACA, Brenda; DE CARVALHO, Sandra. When perfect means plural: the present perfect in Northeastern Brazilian Portuguese. In: CABREDOHOFHERR, P.; LACA, B. (eds.). Layers of aspect. Stanford: CSLI Publications, 2010. p. 67-100.

DAVIDSON, D. The Logical Form of Action Sentences', In: RESCHER, N. (ed.). The Logic of Decision and Action. Pittsburgh: University of Pittsburgh Press, [19??]. p. 81-95.

GIORGI, Alessandra; PIANESI, Fabio. Tense and aspect: from semantics to morphosyntax. New York: Oxford University Press, 1997. 
HOPPER, P. John. On some principles of grammaticalization. In: TRAUGOTT, E.C.; B. HEINE, B. (eds.). Approaches to grammaticalization. Amsterdam/Philadelphia: John Benjamins, 1991. p. 17-36.

HOPPER, Paul; TRAUGOTT, Elisabeth-Cross. Grammaticalization, Cambridge: Cambridge University Press, 1993.

ILARI, Rodolfo. Notas para uma semântica do passado composto em português. Revista Letras, n. 55, p. 129-152, 2001.

JACINTO, Joana. O pretérito perfeito composto: de onde vem a 'temporaridade'? Comemorações dos 75 anos do CLUL Sessão de Estudantes, 5 de Novembro de 2007. 2007. Available at: <http://www.clul.ul.pt/english/artigos/jacinto_joana.pdf $>$.

KLEIN, Wolfgang. The present perfect puzzle. Language, v. 68, n. 3, p. $525-552,1992$.

KLEIN, W. Time in language. London: Routledge, 1994.

LASERSOHN, Peter. Plurality, conjunction and events. Dordrecht: Kluwer, 1995.

LINK, G. The logical analysis of plurals and mass terms: a lattice-theoretical approach. In: BAUERLES, R.; SCHWARZE, C.; STECHOW, A. von. (ed.). Meaning, use and interpretation of language. Berlin: Walter de Gruyter, 1983. p. 303-323.
MOLSING, Karina V. The present perfect: an exercise in the study of events, plurality and aspect. $\mathrm{PhD}$ dissertation. Pontifícia Universidade Católica do Rio Grande do Sul, 2010.

MOLSING, Karina V.; IBAÑOS, Ana Maria Tramunt. Time and TAME in Language. Newcastle upon Tyne: Cambridge Scholars Publishing, 2013.

SCHEIN, Barry. Plurals and events. Cambridge: The MIT Press, 1993.

SCHEIN, Barry. Events and the semantic content of thematic relations. In: PREYER, Gerhard; PETER, Georg (eds.). Logical form, language \& semantic content: on contemporary developments in the philosophy of language \& linguistics. Oxford: Oxford University Press, 1999.

SCHMITT, Cristina. Cross-linguistic variation and the present perfect: the case of portuguese. Natural Language and Linguistic Theory, v. 9, p. 403-453, 2001.

SUTER, Alfred. Das portugiesische pretérito perfeito composto. Bern: Francke, 1984.

Recebido: 02 de fevereiro de 2016. Aprovado: 02 de junho de 2016.

Contato: karina.molsing@pucrs.br 